\title{
46. RARE MIDDLE MIOCENE TO HOLOCENE PLANKTONIC FORAMINIFERAL REMAINS WITH SPECIAL EMPHASIS ON GLOBOROTALIA IN SEDIMENTS OF THE PERU CONTINENTAL SLOPE: SITES 683, 684, 686, 687, AND 6881
}

\author{
Georgette Glaçon, ${ }^{2}$ Patrick De Wever, ${ }^{3}$ and Jacques Bourgois ${ }^{4}$
}

\section{INTRODUCTION}

Species of Globorotalia are among the most dissolutionresistant planktonic foraminifers in sediments of the inner wall of the Middle America Trench; parts of their phylogenetic history have been recognized in sediments of Leg 107 (Glaçon and Bourgois, 1985). These species can be integrated into the biostratigraphic scheme on the basis of calcareous and siliceous nannoplankton and calibrated on the basis of paleomagnetism (Keller, 1980, 1981; Keller et al., 1982; Barron and Keller, 1982). Data compiled for this data report extend to the southern area of occurrence of Globorotalia species.

About 250 sediment samples were collected on board JOIDES Resolution and examined as follows: $20-\mathrm{cm}^{3}$ samples were dried for $8 \mathrm{hr}$ at $60^{\circ} \mathrm{C}$, weighed, and then washed through sieves of $0.5,0.2,0.125$, and $0.063 \mathrm{~mm}$ mesh size. The residues were dried and reweighed. The abundance of planktonic foraminifers counted is reported as numbers of specimens per weight of the original sample.

\section{SITE 683}

Hole 683A (Position: $9^{\circ} 01.69^{\prime} \mathrm{S}, 8^{\circ} 24.40^{\prime} \mathrm{W}$; Water depth [rig floor]: $\mathbf{3 0 8 2 . 3} \mathbf{m}$ )

Forty-one samples were studied from Cores 112-683A-13X to $112-683 A-45 X$; planktonic foraminifers occur only in Sample $112-683 \mathrm{~A}-41 \mathrm{X}-4,81-85 \mathrm{~cm}$. Benthic foraminifers occur in the following samples: $112-683 \mathrm{~A}-13 \mathrm{X}-1,49-52 \mathrm{~cm} ; 112-683 \mathrm{~A}-$ $29 \mathrm{X}-1$, 96-100 cm; 112-683A-32X-1, 47-51 cm; 112-683A$39 \mathrm{X}-1,126-129 \mathrm{~cm}$; $112-683 \mathrm{~A}-39 \mathrm{X}-2,19-23 \mathrm{~cm} ; 112-683 \mathrm{~A}-$ $41 \mathrm{X}-4,81-85 \mathrm{~cm}$; and $112-683 \mathrm{~A}-44 \mathrm{X}-3,107-110 \mathrm{~cm}$. The following samples were barren of foraminifers: 112-683A$14 \mathrm{X}-1,70-74 \mathrm{~cm} ; 112-683 \mathrm{~A}-15 \mathrm{X}-4,100-103 \mathrm{~cm}$; $112-683 \mathrm{~A}-$ $16 \mathrm{X}-2,31-35 \mathrm{~cm} ; 112-683 \mathrm{~A}-17 \mathrm{X}-1,41-45 \mathrm{~cm} ; 112-683 \mathrm{~A}-$ $18 \mathrm{X}-2$, $51-53 \mathrm{~cm} ; 112-683 \mathrm{~A}-18 \mathrm{X}-3,52-56 \mathrm{~cm} ; 112-683 \mathrm{~A}-$ 19X-1, 9-11 cm; 112-683A-20X-1, 13-17 cm; 112-683A-21X1, 37-34 cm; 112-683A-22X-CC, 6-9 cm; 112-683A-23X-1, $34-38 \mathrm{~cm} ; 112-683 \mathrm{~A}-24 \mathrm{X}-1,53-56 \mathrm{~cm} ; 112-683 \mathrm{~A}-25 \mathrm{X}-5,37-$ $40 \mathrm{~cm} ; 112-683 \mathrm{~A}-26 \mathrm{X}-1,47-49 \mathrm{~cm} ; 112-683 \mathrm{~A}-27 \mathrm{X}-1,88-90$ $\mathrm{cm} ; 112-683 \mathrm{~A}-31 \mathrm{X}-1,65-67 \mathrm{~cm} ; 112-683 \mathrm{~A}-33 \mathrm{X}-4,95-99 \mathrm{~cm}$; 112-683A-34X-1, 68-71 cm; 112-683A-34X-3, 130-132 cm; $112-683 \mathrm{~A}-35 \mathrm{X}-3,42-45 \mathrm{~cm}$; 112-683A-36X-2, 124-127 cm; $112-683 \mathrm{~A}-36 \mathrm{X}-4,123-127 \mathrm{~cm} ; 112-683 \mathrm{~A}-37 \mathrm{X}-1,120-124 \mathrm{~cm}$; $112-683 \mathrm{~A}-37 \mathrm{X}-4,41-45 \mathrm{~cm}$; $112-683 \mathrm{~A}-38 \mathrm{X}-\mathrm{CC}, 14-20 \mathrm{~cm}$;

\footnotetext{
${ }^{1}$ Suess, E., von Huene, R., et al., 1990. Proc. ODP, Sci. Results, 112: College Station, TX (Ocean Drilling Program).

2 Laboratoire de Stratigraphie et de Paléoécologie, Université de Provence, 3 Place Victor Hugo, 13331 Marseille Cedex 3, France.

${ }^{3}$ Laboratoire de Stratigraphie, Université Pierre et Marie Curie, 4 Place Jussieu, 75230 Paris Cedex 05, France.

${ }^{4}$ Département de Géotectonique, Université Pierre et Marie Curie, 4 Place Jussieu, 75230 Paris Cedex 05, France.
}

112-683A-39X-4, 126-129 cm; 112-683A-39X-5, 62-66 cm; 112-683A-40X-1, 89-93 cm; 112-683A-40X-3, 89-93 cm; 112$683 \mathrm{~A}-41 \mathrm{X}-1,36-39 \mathrm{~cm} ; 112-683 \mathrm{~A}-42 \mathrm{X}-1,10-14 \mathrm{~cm} ; 112-$ $683-44 X-3,107-109 \mathrm{~cm} ; 112-683 \mathrm{~A}-44 \mathrm{X}-6,108-112 \mathrm{~cm} ; 112-$ $683 \mathrm{~A}-45 \mathrm{X}-1,141-144 \mathrm{~cm}$; and 112-683A-45X-5, 141-144 cm.

\section{Sample 112-683A-41X-4, 81-85 cm (378.0 mbsf)}

The weight of the original sample was $8.67 \mathrm{~g}$; no particle was larger than $0.5 \mathrm{~mm}$; at $0.017 \mathrm{~g}$, no larger than $0.2 \mathrm{~mm}$; at $0.007 \mathrm{~g}$, no larger than $0.125 \mathrm{~mm}$, and at $0.022 \mathrm{~g}$, no larger than $0.063 \mathrm{~mm}$. Only two dextral Globorotalia (Fohsella) peripheroacuta Blow and Banner occur on the $0.125-\mathrm{mm}$ sieve, in association with three Globigerina quinqueloba Natland. A few smaller $G$. quinqueloba are present on the $0.063-\mathrm{mm}$ sieve. $G$. (F.) peripheroacuta is an index fossil of short duration in Zones N10-N11 (Blow, 1969). This species is well represented at Site 568 on the upper slope of the Middle America Trench.

\section{Hole 683B (Position, $9^{\circ} 01.59^{\prime} \mathrm{S}, 8^{\circ} 24.26^{\prime} \mathrm{W}$; Water depth [rig floor]: $3082.0 \mathrm{~m}$ )}

Ten samples were collected from Cores 112-683B-1H to 112-683B-8H; planktonic foraminifers occur only in Sample 112-683B-1H-1, 84-88 cm.

Benthic foraminifers occur in the following samples: 112683B-3H-1, 54-58 cm; 112-683B-5H-1, 64-67 cm; 112-683B$5 \mathrm{H}-\mathrm{CC}, 1-4 \mathrm{~cm}$; and $112-683 \mathrm{~B}-6 \mathrm{H}-3,53-56 \mathrm{~cm}$.

The following samples are barren of foraminifers: 112683B-2H-1, 73-78 cm; 112-683B-6H-1, 124-126 cm; 112683B-7H-1, 94-97 cm; 112-683B-7H-3, 20-23 cm; and 112683B-8H-CC, 40-44 cm.

\section{Sample 112-683B-1H-1, 84-88 cm (403.36 mbsf)}

The weight of the original sample was $8.07 \mathrm{~g}$; the fraction larger than $0.5 \mathrm{~mm}=0.037 \mathrm{~g}$; at $0.015 \mathrm{~g}$, no fraction larger than $0.2 \mathrm{~mm}$; at $0.019 \mathrm{~g}$, no larger than $0.125 \mathrm{~mm}$, and at 0.023 $\mathrm{g}$, no larger than $0.063 \mathrm{~mm}$. No Globorotalia are present. The only planktonic foraminifer species that occur in the fraction smaller than $0.5 \mathrm{~mm}$ are Globigerinoides quadrilobatus (d'Orbigny), common; G. sacculifer (Brady), rare; G. triloba ( $\mathrm{Re}-$ uss), rare; and Dentoglobigerina altispira (Cushman and Jarvis), rare. This assemblage gives an imprecise age for the Miocene-Pliocene (Zones N6-N21; Blow, 1969). Only one Orbulina universa (d'Orbigny) larger than $0.2 \mathrm{~mm}$ is present; this still living species appears in Zone N9 of Blow (1969).

\section{SITE 684}

Hole 684A (Position: $8^{\circ} 59.59 ' \mathrm{~S}$, 79W54.35' W; Water depth [rig floor]: $\mathbf{4 3 6 . 5} \mathrm{m}$ )

Fifteen samples were collected from Cores $112-684 \mathrm{~A}-1 \mathrm{H}$ to 112-684A-9H. Planktonic foraminifers occur only in Samples 
112-684A-1H-3, 90-94 cm, and 112-684A-2H-3, 96-100 cm. Benthic foraminifers occur in the following samples: 112 $684 \mathrm{~A}-1 \mathrm{H}-3,90-94 \mathrm{~cm} ; 112-684 \mathrm{~A}-2 \mathrm{H}-3,96-100 \mathrm{~cm}$; $112-684 \mathrm{~A}-$ $3-1,96-100 \mathrm{~cm} ; 112-684 \mathrm{~A}-4 \mathrm{H}-2,90-94 ; 112-684 \mathrm{~A}-5 \mathrm{H}-2,48$ $52 \mathrm{~cm} ; 112-684 \mathrm{~A}-5 \mathrm{H}-4,48-52 \mathrm{~cm} ; 112-684 \mathrm{~A}-6 \mathrm{H}-2,106-109$ cm; $112-684 \mathrm{~A}-6 \mathrm{H}-4,98-102 \mathrm{~cm} ; 112-684 \mathrm{~A}-8 \mathrm{H}-1,29-33 \mathrm{~cm}$; 112-684A-8H-4, 74-78 cm; and 112-684A-9H-1, 64-68 cm.

The following samples are barren of foraminifers: 112$684 \mathrm{~A}-3 \mathrm{H}-3$, 96-100 cm; 112-684A-4H-4, 90-94 cm; $112-$ 684A-7H-1, 62-64 cm; and 112-684A-7H-4, 78-82 cm.

\section{Sample 112-684A-1H-3, 90-94 cm (3.92 mbsf)}

The weight of the original sample was $8.04 \mathrm{~g}$; the fraction larger than $0.5 \mathrm{~mm}=0.191 \mathrm{~g}$; fraction larger than $0.2 \mathrm{~mm}=$ $0.030 \mathrm{~g}$; fraction larger than $0.125 \mathrm{~mm}=0: 011 \mathrm{~g}$; fraction larger than $0.063 \mathrm{~mm}=0.100 \mathrm{~g}$.

Only 12 specimens of Neogloboquadrina dutertrei (d'Orbigny) occur; two of these are larger than $0.2 \mathrm{~mm}$; the others are a little smaller. All are dextrally coiled and of the subcretacea phenotype of Lomnicki, which means without umbilical plates. These just allow us to conclude that this sample is of the Quaternary Neogloboquadrina eggeri Zone of Thompson and Sciarrillo (1978) in Zone N21 of Blow (1969) to the Holocene.

\section{Sample 112-684A-2H-3, 96-100 cm (9.28 mbsf)}

The weight of the original sample was $12.96 \mathrm{~g}$; the fraction larger than $0.5 \mathrm{~mm}=0.346 \mathrm{~g}$; the fraction larger than $0.2 \mathrm{~mm}$ $=7.720 \mathrm{~g}-\mathrm{a}$ fantastic accumulation when compared with the previously studied sample. The fraction larger than $0.125 \mathrm{~mm}$ $=1.728 \mathrm{~g}$; fraction larger than $0.063 \mathrm{~mm}=1.183 \mathrm{~g}$.

The planktonic assemblage is a composite melange of cool and warm species: Neogloboquadrina pachyderma (Ehrenberg), some sinistrally coiled; Globigerina bulloides and G. quinqueloba are common in the $0.125 \mathrm{~mm}$ fraction; and in the $0.2-\mathrm{mm}$ fraction, common Neogloboquadrina dutertrei occur in association with Orbulina universa. These are predominantly dextrally coiled, and some have an umbilical plate. In the fraction larger than $0.5 \mathrm{~mm}$, five Orbulina universa can be seen with two Sphaeroidinella dehiscens (Parker and Jones). The age is Quaternary.

\section{SITE 686}

Hole 686A (Position: $1^{\circ} 28.81^{\prime} \mathrm{S}, 7^{\circ} 53.49^{\prime} \mathrm{W}$; Water depth [rig floor]: $\mathbf{4 5 7 . 3} \mathrm{m}$ )

Thirty-two samples were collected from Cores 112-686A$1 \mathrm{H}$ to $112-686 \mathrm{~A}-23 \mathrm{X}$. Planktonic foraminifers occur in only two of them: 112-686A-16X-7, 46-50 cm, and 112-686A$21 \mathrm{X}-5,106-110 \mathrm{~cm}$. Benthic foraminifers occur in the following samples: $112-686 \mathrm{~A}-8 \mathrm{H}-1,95-98 \mathrm{~cm} ; 112-686 \mathrm{~A}-12 \mathrm{X}-1$, 97-101 cm ; 112-686A-12X-2, 38-42 cm; 112-686A-14X-2, 73-77 cm; 112-686A-15X-8, 24-28 cm; 112-686A-16X-7, 46$50 \mathrm{~cm} ; 112-686 \mathrm{~A}-21 \mathrm{X}-5,106-110 \mathrm{~cm} ; 112-686 \mathrm{~A}-23 \mathrm{X}-2,100-$ $103 \mathrm{~cm} ; 112-686 \mathrm{~A}-23 \mathrm{X}-4,100-103 \mathrm{~cm}$. The following samples were barren of foraminifers: $112-686 \mathrm{~A}-1 \mathrm{H}-3,90-94 \mathrm{~cm} ; 112-$ $686 \mathrm{~A}-2 \mathrm{H}-1$, 96-100 cm; $112-686 \mathrm{~A}-2 \mathrm{H}-3$, 96-100 cm; $112-$ $686 \mathrm{~A}-3 \mathrm{H}-3,98-102 \mathrm{~cm}$; $112-686 \mathrm{~A}-4 \mathrm{H}-1,94-98 \mathrm{~cm}$; $112-686 \mathrm{~A}-$ $5 \mathrm{H}-1,95-98 \mathrm{~cm} ; 112-686 \mathrm{~A}-5 \mathrm{H}-3,94-98 \mathrm{~cm} ; 112-686 \mathrm{~A}-6 \mathrm{H}-3$, 95-99 cm; 112-686A-7H-1, 96-100 cm; 112-686A-9H-1, 105$109 \mathrm{~cm} ; 112-686 \mathrm{~A}-10 \mathrm{X}-1,107-111 \mathrm{~cm} ; 112-686 \mathrm{~A}-10 \mathrm{X}-3,70-74$ $\mathrm{cm}$; 112-686A-11X-3, 53-57 cm; 112-686A-11X-5, 54-58 cm; 112-686A-13X-3, 105-109 cm; 112-686A-16X-6, 46-50 cm; 112-686A-17X-1, 97-101 cm; 112-686A-18X-1, 107-111 cm; 112-686A-18X-3, 131-135 cm; 112-686A-19X-4, 74-78 cm; 112-686A-19X-6, 74-78 cm; 112-686A-20X-2, 124-128 cm; and $112-686 \mathrm{~A}-20 \mathrm{X}-4,124-128 \mathrm{~cm}$.
Sample 112-686B-16X-7, 46-50 cm (138.18 mbsf)

The weight of the original sample was $10.95 \mathrm{~g}$; fraction larger than $0.5 \mathrm{~mm}=0.038 \mathrm{~g}$; fraction on $0.25-\mathrm{mm}$ sieve $=$ $0.030 \mathrm{~g}$; on $0.125-\mathrm{mm}$ sieve $=0.060 \mathrm{~g}$, and on $0.063-\mathrm{mm}$ sieve $=0.071 \mathrm{~g}$.

Only one Neogloboquadrina dutertrei, dextrally coiled, was found.

\section{Sample 112-686B-21X-5, 106-110 cm (184.26 mbsf)}

The weight of the original sample was $16.47 \mathrm{~g}$; the fraction larger than $0.5 \mathrm{~mm}=0.109 \mathrm{~g}$; fraction on $0.2-\mathrm{mm}$ sieve $=$ 0.065 ; on $0.125-\mathrm{mm}$ sieve $=0.021 \mathrm{~g}$; and on $0.063-\mathrm{mm}$ sieve $=$ $0.190 \mathrm{~g}$.

Only four Neogloboquadrina dutertrei were found, which seems to indicate that this level is still in the Quaternary $(N$. eggeri Zone of Thompson and Sciarrillo, 1978).

\section{Hole 686B (Position: $1^{\circ} 28.81^{\prime} \mathrm{S}, 7^{\circ} 53.49^{\prime} \mathrm{W}$; Water depth [rig floor]: $\mathbf{4 5 8 . 3} \mathrm{m}$ )}

Fifteen samples were collected from Cores 112-686B-23X to $112-686 \mathrm{~B}-32 \mathrm{X}$; planktonic foraminifers only occur in Sample 112-686B-26X-3, 96-100 cm. Benthic foraminifers occur in the following samples: $112-686 \mathrm{~B}-23 \mathrm{X}-3,108-112 \mathrm{~cm} ; 112-$ 686B-24X-3, 98-102 cm; 112-686B-25X-1, 87-91 cm; 112686B-26X-1, 96-100 cm; 112-686B-26X-3, 96-100 cm; 112686B-28X-3, 46-50 cm; 112-686B-30X-6, 15-17 cm; 112686B-32X-1, 61-65 cm; and 112-686B-32X-2, 72-76 cm. The following samples are barren of foraminifer: 112-686B-23X-1, $108-102 \mathrm{~cm}$; 112-686B-24-1,98-10 25X-3, 87-91 cm; 112686B-28X-5, 46-50 cm; 112-686B-29X-3, 18-22 cm; and 112686B-30X-3, $15-17 \mathrm{~cm}$.

\section{Sample 112-686B-26X-3, 96-100 cm (240.48 mbsf)}

The weight of the original sample $=9.08 \mathrm{~g}$; the fraction larger than $0.5 \mathrm{~mm}=0.122 \mathrm{~g}$; fraction on $0.2-\mathrm{mm}$ sieve $=$ $0.056 \mathrm{~g}$; on $0.125-\mathrm{mm}$ sieve $=0.054 \mathrm{~g}$, and on $0.063-\mathrm{mm}$ sieve $=0.059 \mathrm{~g}$.

There were 12 Globigerina quinqueloba smaller than $0.2 \mathrm{~mm}$.

\section{SITE 687}

\section{Hole 687A (Position: 12 $^{\circ} 51.78^{\prime} \mathrm{S} ; 7^{\circ}{ }^{\circ} 59.43^{\prime} \mathrm{W}$;} Water depth [rig floor]: $317.3 \mathrm{~m}$ )

Twenty-four samples were collected; planktonic foraminifers occur in only one: $112-687 \mathrm{~A}-21 \mathrm{X}-1,125-129 \mathrm{~cm}$.

Benthic foraminifers occur in the following samples: 112-687A-3H-1, 89-93 cm; 112-687A-3H-3, 58-62 cm; 112$687 \mathrm{~A}-13 \mathrm{H}-2,58-62 \mathrm{~cm} ; 112-687 \mathrm{~A}-14 \mathrm{X}-1,132-136 \mathrm{~cm} ; 112-$ 687A-15X-1, 139-143 cm; 112-687A-17X-1, 96-100 cm; 112687A-17X-3, 93-97 cm; 112-687A-18X-1, 129-133 cm; 112687A-19X-1, 96-100 cm; 112-687A-19X-3, 96-100 cm; 112$687 \mathrm{~A}-21 \mathrm{X}-1,125-129 \mathrm{~cm}$; and 112-687A-22X-3, 132-136 cm.

The following samples are barren of foraminifers: 112$687 \mathrm{~A}-1 \mathrm{H}-3,30-34 \mathrm{~cm} ; 112-687 \mathrm{~A}-1 \mathrm{H}-5,30-34 \mathrm{~cm} ; 112-$ $687 \mathrm{~A}-2 \mathrm{H}-3,72-76 \mathrm{~cm} ; 112-687 \mathrm{~A}-4 \mathrm{H}-1,72-76 \mathrm{~cm} ; 112-$ $687 \mathrm{~A}-4 \Omega-3,72-76 \mathrm{~cm} ; 112-687 \mathrm{~A}-5 \mathrm{H}-1,105-109 \mathrm{~cm} ; 112-$ 687 A-5H-4, 105-109 cm; 112-687A-6H-1, 108-112 cm; $112-$ 687A-6H-3, 108-112 cm; 112-687A-7H-2, 20-24 cm; 112$687 \mathrm{~A}-13 \mathrm{H}-6,52-56 \mathrm{~cm}$; and 112-687A-21X-3, $125-129 \mathrm{~cm}$.

\section{Sample 112-687A-21X-1, 125-129 cm (189.27 mbsf)}

The weight of the original sample was $9.74 \mathrm{~g}$; the fraction larger than $0.5 \mathrm{~mm}=1.073 \mathrm{~g}$; fraction on $0.250-\mathrm{mm}$ sieve $=$ $0.982 \mathrm{~g}$; on $0.150-\mathrm{mm}$ sieve $=1.022 \mathrm{~g}$; and on $0.063-\mathrm{mm}$ sieve $=1.279 \mathrm{~g}$. 


\begin{tabular}{|c|c|c|c|c|c|c|c|c|c|c|c|c|c|}
\hline \multirow{2}{*}{$\begin{array}{l}\text { Core, section, } \\
\text { interval }(\mathrm{cm})\end{array}$} & \multirow{2}{*}{$\begin{array}{l}\text { Original } \\
\text { sample }\end{array}$} & & Wei & t $(\mathrm{g})$ & & \multirow[t]{2}{*}{ 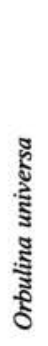 } & \multirow[t]{2}{*}{ 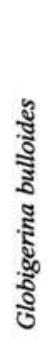 } & \multirow[t]{2}{*}{ 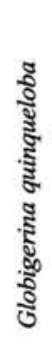 } & \multirow[t]{2}{*}{ 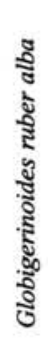 } & \multirow[t]{2}{*}{ 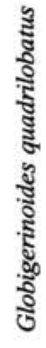 } & \multirow[t]{2}{*}{ 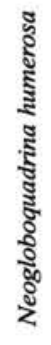 } & \multirow[t]{2}{*}{ 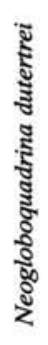 } & \multirow[t]{2}{*}{ 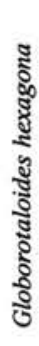 } \\
\hline & & $0.500 \mathrm{~mm}$ & $0.200 \mathrm{~mm}$ & $0.125 \mathrm{~mm}$ & $0.063 \mathrm{~mm}$ & & & & & & & & \\
\hline $112-688 \mathrm{~A}-1 \mathrm{H}-1,96-100$ & 9.46 & 0.006 & 0.035 & 0.033 & 0.284 & & 3 & 8 & & & & & \\
\hline $1 \mathrm{H}-3,96-100$ & 10.18 & 0.006 & 0.036 & 0.062 & 0.084 & & 1 & & & & & & \\
\hline $2 \mathrm{H}-1,96-100$ & 9.93 & 0.000 & 0.079 & 0.027 & 0.500 & & 1 & & & & & & \\
\hline $3 \mathrm{H}-2,50-54$ & 12.47 & 0.076 & 0.045 & 0.013 & 0.083 & 1 & 1 & & & & & & \\
\hline $3 \mathrm{H}-5,51-54$ & 12.38 & 0.051 & 0.059 & 0.069 & 0.093 & & & & & & 4 & 3 & \\
\hline $4 \mathrm{H}-1,119-123$ & 13.78 & 0.000 & 0.049 & 0.038 & 0.227 & & 1 & & & & & & \\
\hline $5 \mathrm{H}-4,56-60$ & 15.78 & 0.026 & 0.070 & 0.080 & 0.236 & & & & & & 2 & 4 & \\
\hline $6 \mathrm{H}-5,74-78$ & 14.31 & 0.000 & 0.040 & 0.027 & 0.093 & & 4 & & & 1 & & 5 & 3 \\
\hline $13 X-3,110-114$ & 17.47 & 0.000 & 0.002 & 0.010 & 0.025 & & & & & & & 1 & \\
\hline $14 X-3,88-92$ & 12.42 & 0.000 & 0.059 & 0.042 & 0.064 & & & & & 2 & & 6 & \\
\hline $17 X-3,95-99$ & 13.72 & 0.000 & 0.023 & 0.032 & 0.094 & & 1 & & & 3 & & & \\
\hline $19 X-3,95-98$ & 15.86 & 0.017 & 0.042 & 0.034 & 0.061 & & & & 1 & 1 & 1 & & \\
\hline
\end{tabular}

Note: Counted by number of specimens per weight of original sample.

Two dextral Neogloboquadrina humerosa (Takayanagi and Saito) and a dextral Neogloboquadrina dutertrei occur in the fraction larger than $0.250 \mathrm{~mm}$; on the $0.150-\mathrm{mm}$ sieve we found a Globigerinoides ruber cyclostomus (Galloway and Wissler). This assemblage, if it is a natural one, gives us an age between the Jaramillo and Olduvai (low Zone N22 of Blow, 1969).

\section{SITE 688}

\section{Hole 688A (Position: $1^{\circ} 32.26^{\prime} \mathrm{S} ; 7^{\circ} 56.57^{\prime} \mathrm{W}$; Water depth [rig floor]: $\mathbf{3 8 3 0 . 3} \mathbf{~ m}$ )}

Forty-seven samples were collected. Only one mold of a Globorotalia "scituliform" occurs in Sample 112-688A-17X$3,95-99 \mathrm{~cm}$. Other rare planktonic foraminifers are scattered in Cores 112-688A-1H, 112-688a-2H, 112-688A-3H, 112$688 \mathrm{~A}-5 \mathrm{H}, 112-688 \mathrm{~A}-6 \mathrm{H}, 112-688 \mathrm{~A}-13 \mathrm{X}, 112-688 \mathrm{~A}-14 \mathrm{X}, 112-$ 688A-17X, and 112-688A-19X (Table 1).

Benthic foraminifers occur in the following samples: $112-688 \mathrm{~A}-1 \mathrm{H}-1,96-100 \mathrm{~cm}$; $112-688 \mathrm{~A}-1 \mathrm{H}-3,96-100 \mathrm{~cm}$; $112-688 \mathrm{~A}-3 \mathrm{H}-2,50-54 \mathrm{~cm} ; 112-688 \mathrm{~A}-3 \mathrm{H}-5,51-54 \mathrm{~cm} ; 112-$ $688 \mathrm{~A}-4 \mathrm{H}-1,119-123 \mathrm{~cm} ; 112-688 \mathrm{~A}-4 \mathrm{H}-3,119-123 \mathrm{~cm} ; 112-$ $688 \mathrm{~A}-5 \mathrm{H}-2,56-60 \mathrm{~cm} ; 112-688 \mathrm{~A}-5 \mathrm{H}-3,105-107 \mathrm{~cm} ; 112-$ $688 \mathrm{~A}-5 \mathrm{H}-4,56-60 \mathrm{~cm} ; 112-688 \mathrm{~A}-7 \mathrm{H}-3,90-94 \mathrm{~cm} ; 112-$ $688 \mathrm{~A}-8 \mathrm{H}-2,82-86 \mathrm{~cm} ; 112-688 \mathrm{~A}-32 \mathrm{X}-5,70-74 \mathrm{~cm} ; 112-$ $688 \mathrm{~A}-33 \mathrm{X}-2,61-65 \mathrm{~cm}$; and $112-688 \mathrm{~A}-37 \mathrm{X}-1,103-107 \mathrm{~cm}$. The following samples are barren of foraminifers: 112$688 \mathrm{~A}-2 \mathrm{H}-3,96-100 \mathrm{~cm} ; 112-688 \mathrm{~A}-6 \mathrm{H}-3,74-78 \mathrm{~cm} ; 112-$ $688 \mathrm{~A}-7 \mathrm{H}-1,90-94 \mathrm{~cm} ; 112-688 \mathrm{~A}-10 \mathrm{H}-2,89-93 \mathrm{~cm} ; 112-$ $688 \mathrm{~A}-11 \mathrm{X}-5,88-92 \mathrm{~cm} ; 112-688 \mathrm{~A}-11 \mathrm{X}-7,88-92 \mathrm{~cm} ; 112-$ $688 \mathrm{~A}-12 \mathrm{X}-1,92-96 \mathrm{~cm} ; 112-688 \mathrm{~A}-13 \mathrm{X}-1,110-114 \mathrm{~cm} ; 112-$ 688A-14X-1, 95-100 cm; 112-688A-15X-1, $14-17 \mathrm{~cm} ; 112-$ 688A-16X-1, 93-97 cm; 112-688A-16X-4, 93-97 cm; 112688A-17X-1, 94-98 cm; 112-688A-18X-1, 110-114 cm; 112$688 \mathrm{~A}-19 \mathrm{X}-1,95-98 ; 112-688 \mathrm{~A}-21 \mathrm{X}-3,960-100 \mathrm{~cm} ; 112-$ 688A-24X-1, 80-83 cm; 112-688A-26X-1, 53-57 cm; $112-$ 688A-27X-1, 32-36 cm; 112-688A-28X-1, $105-109 \mathrm{~cm} ; 112-$ 688A-29X-2, 42-46 cm; 112-688A-30X-1, 59-63 cm; 112$688 \mathrm{~A}-31 \mathrm{X}-1,15-18 \mathrm{~cm} ; 112-688 \mathrm{~A}-32 \mathrm{X}-4,70-74 \mathrm{~cm} ; 112-$ $688 \mathrm{~A}-35 \mathrm{X}-5,130-134 \mathrm{~cm}$; and $112-688 \mathrm{~A}-36 \mathrm{X}-4,119-123$ $\mathrm{cm}$.
The lower Neogloboquadrina dutertrei Zone is recorded in Core 112-688A-14X-3, 88-92 cm, in the Quaternary, but a problem exists with the upper $N$. humerosa Zone in Core 112-688A-3H (Table 1), which is reworked, or the zone occurs in more recent levels here than in samples of the Middle America Trench (Leg 84), or perhaps they are more recent convergent morphotypes.

\section{Hole 688E (Position: ${ }^{\circ} 32.28^{\prime} \mathrm{S}, \mathbf{7 8}^{\circ} 56.65^{\prime} \mathrm{W}$; Water depth [rig floor]: $\mathbf{3 8 3 6 . 3} \mathbf{~ m}$ )}

All 33 samples collected were barren of planktonic foraminifers. Benthic foraminifers occur in three samples: 112688E-12X-1, 104-108 cm; $112-688 \mathrm{E}-16 \mathrm{X}-1,95-98 \mathrm{~cm}$; and 112-688E-20X-1, 92-96 cm. The following samples were barren of foraminifers: $112-688 \mathrm{E}-1 \mathrm{H}-1,106-109 \mathrm{~cm} ; 112-$ $688 \mathrm{E}-2 \mathrm{H}-1,19-22 \mathrm{~cm} ; 112-688 \mathrm{E}-3 \mathrm{H}-1,107-111 \mathrm{~cm} ; 112-$ $688 \mathrm{E}-3 \mathrm{H}-3,107-111 \mathrm{~cm} ; 112-688 \mathrm{E}-4 \mathrm{H}-1,96-100 \mathrm{~cm} ; 112-$ $688 \mathrm{E}-4 \mathrm{H}-3,96-100 \mathrm{~cm} ; 112-688 \mathrm{E}-5 \mathrm{H}-3,61-65 \mathrm{~cm} ; 112-688 \mathrm{E}-$ $6 \mathrm{H}-4,62-68 \mathrm{~cm} ; 112-688 \mathrm{E}-7 \mathrm{H}-3,61-67 \mathrm{~cm} ; 112-688 \mathrm{E}-8 \mathrm{H}-6$, $79-83 \mathrm{~cm} ; 112-688 \mathrm{E}-9 \mathrm{H}-2,55-59 \mathrm{~cm} ; 112-688 \mathrm{E}-10 \mathrm{X}-3,70-74$ cm; 112-688E-4X-1, 109-113 cm; 112-688E-14X-3, 109-113 $\mathrm{cm} ; 112-688 \mathrm{E}-15 \mathrm{X}-1,89-92 \mathrm{~cm}$; 112-688E-19X-1, 40-44 cm; 112-688E-23X-3, 134-138 cm; 112-688E-24X-1, 144-147 cm; $112-688 \mathrm{E}-25 \mathrm{X}-1,95-98 \mathrm{~cm} ; 112-688 \mathrm{E}-26 \mathrm{X}-1,95-98 \mathrm{~cm} ; 112-$ $688 \mathrm{E}-30 \mathrm{X}-1,72-76 \mathrm{~cm} ; 112-688 \mathrm{E}-34 \mathrm{X}-2,63-66 \mathrm{~cm} ; 112-$ $688 \mathrm{E}-35 \mathrm{X}-1,110-114 \mathrm{~cm} ; 112-688 \mathrm{E}-36 \mathrm{X}-3,52-56 \mathrm{~cm} ; 112-$ 688E-38X-2, 133-137 cm; 112-688E-39X-1, 144-147 cm; 112$688 \mathrm{E}-42 \mathrm{X}-1,128-131 \mathrm{~cm} ; 112-688 \mathrm{E}-43 \mathrm{X}-1,123-126 \mathrm{~cm} ; 112-$ 688E-43X-3, 131-134; and 112-688E-44X-1, 109-113 cm.

\section{REFERENCES}

Barron, J. A., and Keller, G., 1982. Widespread Miocene deep-sea hiatuses: coincidence with periods of global cooling. Geology, 10:577-581.

Blow, W. H., 1969. Late middle Eocene to Recent planktonic foraminiferal biostratigraphy. In Renz, H. H., and Brönniman, P. (Eds.), Proc. Int. Conf. Planktonic Microfossils, 1:199-422.

Glaçon, G., and Bourgois, J., 1985. Upper Oligocene to Recent planktonic foraminiferal remains in sediments of the inner wall of the Middle America Trench with special emphasis on Globorota- 
lia. In von Huene, R., Aubouin, J., et al., Init. Repts. DSDP, 84: Washington (U.S. Govt. Printing Office), 473-513.

Keller, G., 1980. Middle to late Miocene datum levels and paleoceanography of the north and southeastern Pacific Ocean. Mar. Micropaleontol., 5:249-281.

, 1981. The genus Globorotalia in the early Miocene of the equatorial and northwestern Pacific. J. Foraminifer. Res., 11:118132.

Keller, G., Barron, J. A., and Burckle, L. H., 1982. North Pacific late Miocene correlations using microfossils, stable isotopes, percent $\mathrm{CaCO}_{3}$ and magnetostratigraphy. Mar. Micropaleontol., 7:327-357.
Thompson, P. R., and Sciarrillo, J. R., 1978. Planktonic foraminiferal biostratigraphy in the equatorial Pacific. Nature, 276:29-33.

Date of initial receipt: 26 July 1988

Date of acceptance: 11 October 1989

Ms 112B-185 